\title{
The effect of n-acetylcysteine on hepatic histomorphology during hypothermic preservation
}

\author{
Patricia Sayuri Risso ${ }^{\mathrm{I}}$, Marcia Kiyomi Koike ${ }^{\mathrm{II}}$, Marcos de Souza Abrahão ${ }^{\mathrm{III}}$, Natalie Chaves Ferreira ${ }^{\mathrm{IV}}$, Edna Frasson de Souza \\ Monterov $^{\mathrm{V}}$
}

DOI: http://dx.doi.org/10.1590/S0102-86502014001700006

IScientific Initiation student at University City of São Paulo (UNICID). São Paulo, Brazil. Responsible for technical procedures, data acquisition, analysis and interpretation of data and manuscript writing.

IIPhD, Laboratory of Emergency Medicine (LIM-51), Medical School of the São Paulo University (FMUSP). São Paulo, Brazil. Interpretation of data, histopathological, statistical analysis and critical revision.

IIIPhD, Paulista Medicine School of the Federal University of São Paulo (EPM-UNIFESP). São Paulo, Brazil. Responsible for data acquisition, analysis, and interpretation of data.

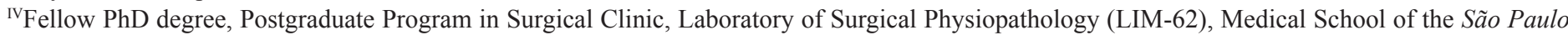
University (FMUSP). São Paulo, Brazil. Responsible for interpretation of data and manuscript writing.

${ }^{v}$ PhD, Associate Professor, Department of Surgery, Laboratory of Surgical Physiopathology (LIM 62), Medical School of the São Paulo University (FMUSP). São Paulo, Brazil. Associate Professor, Operative Technique and Experimental Surgery Division, Department of Surgery, Federal University of São Paulo (UNIFESP). São Paulo, Brazil. Tutor. Responsible for conception, design, intellectual and scientific content of the study. Final approval of the version to be published.

\section{ABSTRACT}

PURPOSE: To evaluate the NAC effects on liver hypothermic preservation at different time intervals.

METHODS: For this, we used livers of male Wistar rats weighing between 250 and 300g, undergoing preservation in Ringer solution at $4^{\circ} \mathrm{C}$ for up to 24 hours. Tissue samples were obtained at four moments of preservation for histological examination by hematoxylin and eosin staining: T0 = beginning of preservation, T12 = 12 hours, T18 $=18$ hours and T24 = 24 hours. Will be analyzed vacuolation, hepatic apoptosis by optical microscopy and parenchymal.

RESULTS: The results showed a progressive increase in hepatic injury in both groups and showed that NAC was effective at T0. The parenchyma preservation was better in the NAC group and no difference when vacuolization of the cells.

CONCLUSION: Hypothermic preservation, over time, causes changes in the hepatic parenchyma with increased apoptosis, loss of architecture, vacuolization, culminating in severe injury. The administration of $\mathrm{N}$-acetylcysteine protects against preservation liver injury. Key words: Acetylcysteine. Liver. Hypothermia. Organ Preservation. Rats. 


\section{Introduction}

In organ transplantation are well-established strategies to maintain morphological aspects and graft function. These strategies include hemodynamic stability of donor, previous health status, absence of diseases. In addition, interventions at the time of the donor operation, such as hypothermia, preservation solution and use of substances, may modulate or ischemic and/or reperfusion injury.

The success of organ transplantation depends on, in part, how it has been preserved. The form of preservation is essential to ensure the viability of the graft during storage, and will determine its functioning post-transplantation. One of the strategies used in organ transplants to minimize the injury caused by ischemia is hypothermia. It decreases the cellular components degradation, which is essential for cell viability, enzymatic reactions speed and cell death occurrence, also prevents intracellular acidosis ${ }^{1-3}$. This cold-induced down regulation of the metabolism protects the graft against reactive species of oxygen, during reperfusion ${ }^{2,4}$.

The ischemia occurs when there is a reduction or interruption of blood flow, leading to oxygen deprivation, activation anaerobic metabolism to obtain energy and inducing imbalance of electrolyte homeostasis, cellular dysfunction, cellular and interstitial edema, intense formation of free radicals and, posteriorly, cell death by necrosis and/or apoptosis ${ }^{5,6}$.

The use of antioxidants has been proposed as pharmacological strategy to modulate ischemia and reperfusion injury (I/R) injury. The antioxidant is used to decrease reactive oxygen species production during I/R process $^{7-9}$.

The glutathione is an endogenous antioxidant, donating electrons to neutralize and removing free radicals. $\mathrm{N}$-acetylcysteine, which is a thiol precursor of glutathione, is an exogenous antioxidant used to induce elevation of hepatic glutathione. Previous studies have shown that $\mathrm{N}$-acetylcysteine attenuated $\mathrm{I} / \mathrm{R}$ injury on liver and intestine in normothermic conditions $\mathrm{s}^{7,9,10}$. In experimental hepatic ischemic injury, the treatment with the $\mathrm{N}$-acetylcysteine, immediately before ischemia, preserved morphological architecture and tissue functionality during ischemia ${ }^{8-10}$. However, its effect on hypothermic liver preservation has not been studied.

The aim of this study was evaluate the effect of $\mathrm{N}$-acetylcysteine on hepatic histomorphology during different intervals of hypothermic preservation.

\section{Methods}

Adult male Wistar rats, weighing 250 to 300 g, were individually caged in a temperature and $12 \mathrm{~h}$ light-dark cycle controlled room. They were fed a standard chow diet and received water ad libitum. The Research Ethics Commission of the Federal University of São Paulo (Protocol CEP 1817/09), Brazil, approved the experimental protocol. The animals were randomly divided into two groups, according the treatment: saline (Control, $n=6$ ) and $\mathrm{N}$-acetylcysteine (NAC, $\mathrm{n}=6$ ).

\section{Experimental design}

All rats were anesthetized intramuscularly with a solution of ketamine $(60 \mathrm{mg} / \mathrm{kg})$ and xylazine $(10 \mathrm{mg} / \mathrm{kg})$ and received intravenous doses of heparin $(100 \mathrm{U} / 100 \mathrm{~g})$. A median laparotomy was performed to exposed abdominal aorta and vena cava and administration of saline or NAC $(150 \mathrm{mg} / \mathrm{kg})$. After 15 minutes, abdominal aorta was catheterized to Ringer lactate $\left(4^{\circ} \mathrm{C}\right)$ perfusion (10 minutes); vena cava was used to blood and perfusion solution drainage, and liver was collected to $24 \mathrm{~h}$ preservation study ${ }^{18}$.

During the period of preservation, the liver was submersing in a cold Ringer lactate solution $\left(4^{\circ} \mathrm{C}\right)$. Hepatic samples were collected for analysis in follow intervals: immediately after excising (T0), 12h (T12), 18h (T18) and 24h (T24). Samples were fixed in buffered $10 \%$ formalin and embedded in paraffin.

\section{Histomorphology}

Tissue sections were cut in 4 micrometers and were stained by hematoxylin-eosin (H. E.). The analysis of injury degrees were performed under a light microscope by pathologist blinded to the project data. To assess hepatic injury (architectural alteration and vacuolization), four fields per section, sum 5000 micrometers square area (under 100x magnification) were analyzed using a computerized image system (Axiovision release 4.7/05-2008) coupled to optical microscope (Axioskop40).

Necrosis and apoptotic hepatocyte death were evaluated under 400x magnification. Apoptosis was identified by histological criteria include condensed nuclear fragments, nuclei with marginated chromatin, multiple nuclear fragments, a single condensed nucleus, membrane-bound structures containing variable amounts of chromatin and/or cytoplasm and eosinophilic cytoplasm ${ }^{11}$. Fields containing portal spaces and centrolobular veins were not considered. Apoptotic index was calculated by ratio of apoptotic number of hepatocyte and total number of hepatocyte. 


\section{Statistical analysis}

For data analysis, it was used the statistical software SigmaStat for Windows. The $\mathrm{p}<0.05$ or $5 \%$ was considered statistically significant. The descriptive analysis of continuous variable was described by mean \pm standard deviation. For the inferential analyze of hepatic injury degree in sequential intervals, time-points were compared by One-way analysis of variance (ANOVA) complemented by Student-Newman Keuls. To analyze the effects of NAC on liver preservation, groups were compared by Mann-Whitney t-test, in the same time-point.

\section{Results}

The values among different time-points showed

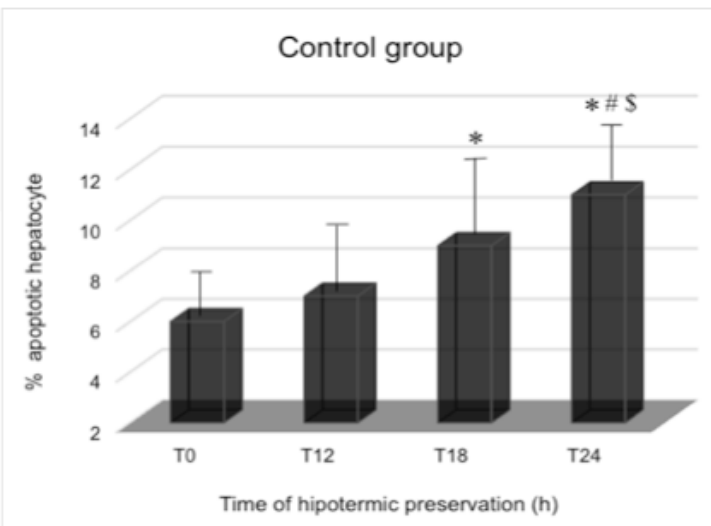

progressive injury, increasing occurrence of apoptotic hepatocyte in both groups (Figure 1). In the Control group, the values at the T24 were significantly higher $(\mathrm{p}<0.05)$ compared to each other time-point. However, in the NAC group, the T24 values were similar to the T12 and T18, and all of them were different from the T0 values $(\mathrm{p}<0.05)$. N-acetylcysteine pretreatment showed important initial reduction of apoptotic hepatocyte $(\mathrm{T} 0=4 \%)$ compared to the control group ( $\mathrm{T} 0=6 \% ; \mathrm{p}<0.05)$.

$\mathrm{N}$-acetylcysteine treatment attenuated the preservation hepatic injury only in the time-point T0, maintaining the tissue architecture and absence of edema compared to control (Figure 2). In the time-point T24, both group showed alterations in the hepatic tissue architecture, but the NAC group showed just a slight tissue injury (Figure 3 ). In both groups, vacuolization occurred after $12 \mathrm{~h}$ of cold preservation.

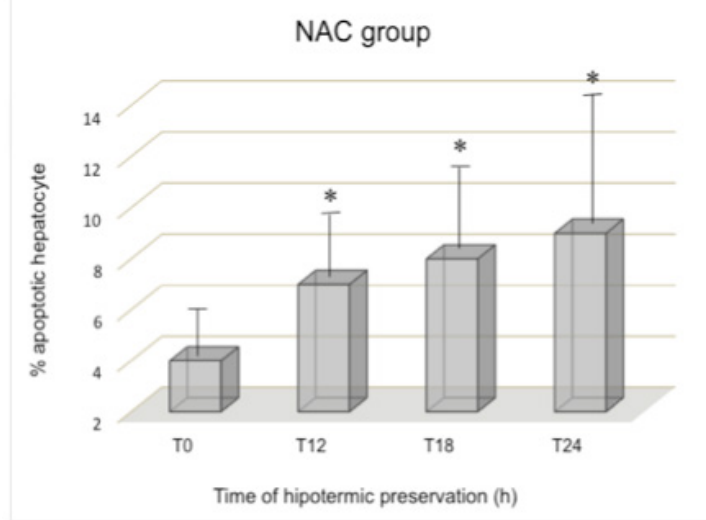

FIGURE 1 - Percentage of apoptotic hepatocyte in different intervals of liver preservation in cold Ringer Lactate. In left panel, observe control liver of rats treated with saline. In right panel, observe NAC liver of rats treated with N-acetylcysteine. Preservation time-points are appointed as T0: 0h; T12: 12h; T18: 18h; T24: 24h; In ANOVA/Student-Newman Keuls post-hoc test: *, p<0.05 vs T0; \#, p<0.05 vs T12; \$, p<0.05 vs T18.
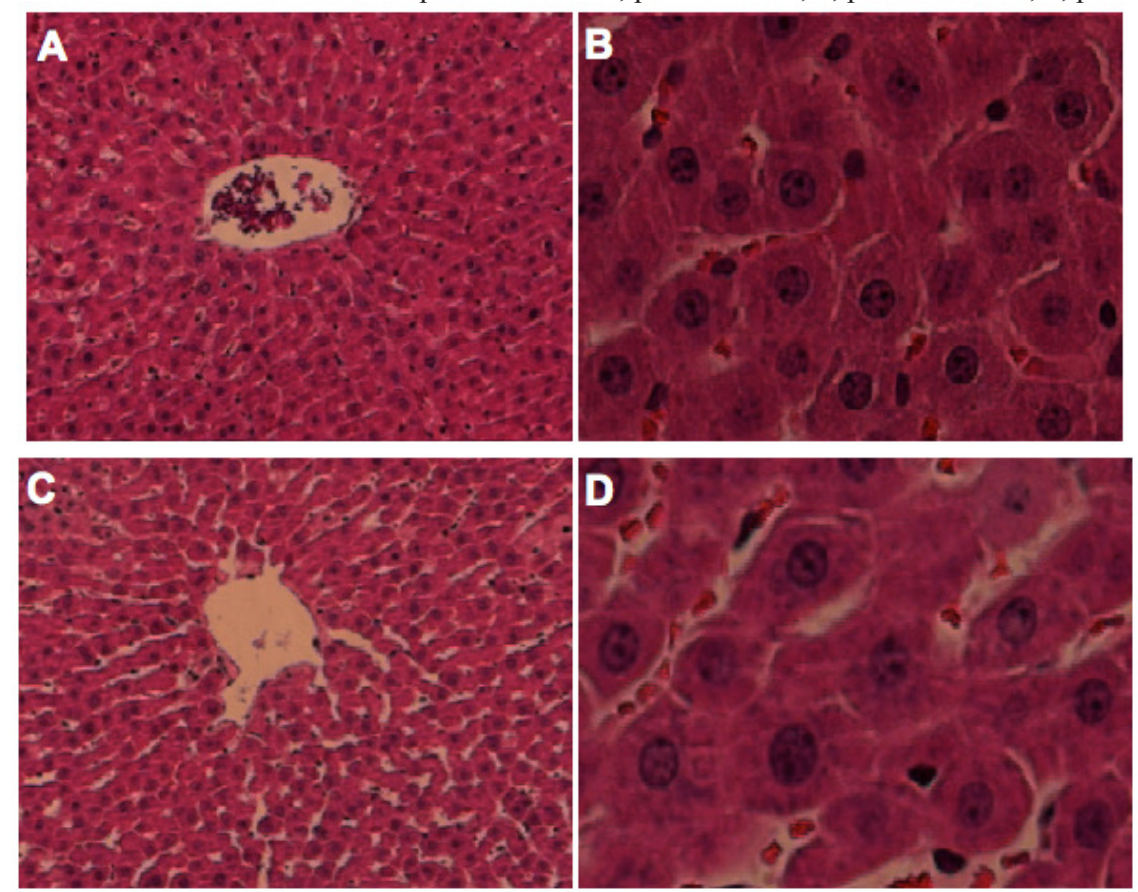

FIGURE 2 - Photomicrographs showing 0h of hypothermic preservation of hepatic tissue in Ringer lactate at $4{ }^{\circ} \mathrm{C}$. The panels A and B illustrate NAC treated group in 100x (left) and 400x (right) magnification. The panels C and D illustrate control group, in same magnification. 

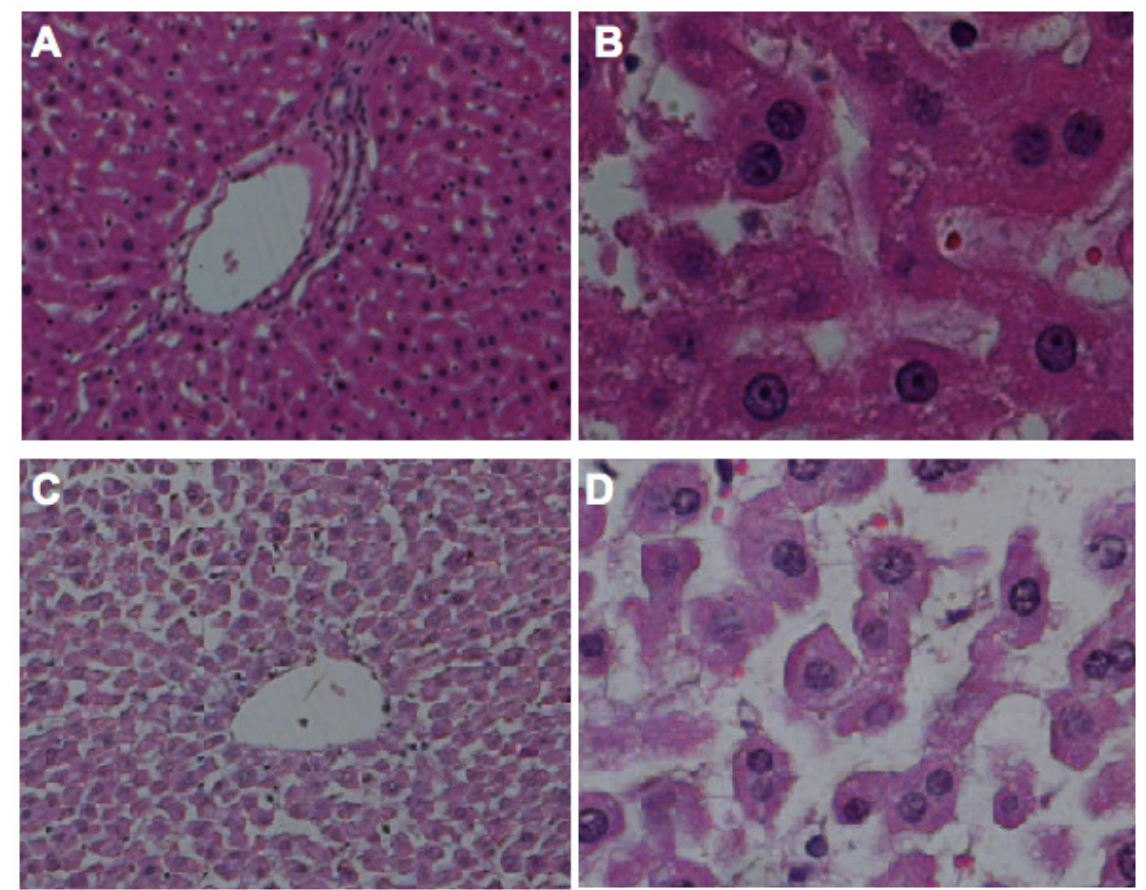

FIGURE 3 - Photomicrographs showing $24 \mathrm{~h}$ of hypothermic preservation of hepatic tissue in Ringer lactate at $4^{\circ} \mathrm{C}$. The panels A and B illustrate NAC treated group in 100x (left) and 400x (right) magnification. The panels $\mathrm{C}$ and D illustrate control group, in same magnification.

\section{Discussion}

The present study showed reduction of apoptosis occurrence in the early phase of liver hypothermic preservation when administered $\mathrm{N}$-acetylcysteine. This effect did not persist during other periods of observation in the study protocol.

The ischemia caused when there is an interruption of the blood supply is well known and the strategies used to minimize this type of injury has been studied. N-acetylcysteine, an antioxidant, has shown beneficial effect on injury $\mathrm{I} / \mathrm{R}$ in the microcirculation and reducing of the interaction between leukocytes and endothelium, but no effect on the activity of cells Kupffer ${ }^{12}$.

$\mathrm{N}$-acetylcysteine in the liver provides protection by inducing increased hepatic glutathione and retain the enzymatic function.

Aspartate aminotransferase, alanine aminotransferase, lactate dehydrogenase and total bilirubin are function liver markers, providing information on liver injury. Studies show that the treatment with $\mathrm{N}$-acetylcysteine reduces these markers and decrease the injury $\mathrm{I} / \mathrm{R}^{3,6}$.

A methodology using light microscopy with hematoxylin and eosin-stained sections has been applied to identify anoikis, nuclear and cytoplasmic condensation, and apoptotic bodies ${ }^{13}$, ${ }^{14}$. The apoptotic index used in this study followed the nuclei and cytoplasm morphological characteristics of hepatocyte apoptosis $^{14-16}$ and results showed significant changes during follow-up. In view of the effects of $\mathrm{N}$-acetylcysteine on the apoptotic index of liver subjected to hypothermic preservation hepatocytes, the future prospect of this study is to confirm the apoptosis by immunohistochemical marking, for example, anticaspase 3 cleaved antibody or TUNEL.

Results of the administration of $\mathrm{N}$-acetylcysteine in this study corroborate the microscopic features reported in previous studies, showing that $\mathrm{N}$-acetylcysteine reduces significantly the degree of hepatic congestion ${ }^{17}$ and preserves parenchimal ${ }^{3}$. However, studies should proceed with reperfusion of the organ to verify more adequately the effect of NAC on liver parenchyma.

\section{Conclusion}

Hypothermic preservation, over time, causes changes in the hepatic parenchyma with increased apoptosis, loss of architecture, vacuolization, culminating in severe injury. The administration of $\mathrm{N}$-acetylcysteine protects against preservation liver injury.

\section{References}

1. Aslami H, Juffermans NP. Induction of hipermetabolic state during critical illness - a new conception in the ICU? Neth J Med. 2010;68(5):190-8. PMID: 20508267.

2. $\mathrm{Xu} \mathrm{H}$, Lee $\mathrm{CY}$, Clemens MG, Zhang JX. Inhibition of TXA synthesis with OKY-046 improves liver preservation by prolonged hypothermic machine perfusion in rats. J Gastroenterol Hepatol. 2008;23 (7 pt2):e212-20. PMID 17645480. 
3. Polderman KH. Mechanisms of action, physiological effects, and complications of hypothermia. Crit Care Med. 2009;37(7Suppl):S186-S202. DOI: 10.1097/CCM.0b013e3181aa5241.

4. Polderman KH. Induced hypothermia and fever control for prevention and treatment of neurological injuries. Lancet. 2008;371(9628):1955-69. DOI: 10.1016/S0140-6736(08)60837-5.

5. Lobb I, Davison M, Carter D, Lan Z, Sener A. P57 Hydrogen sulfide treatment reduces activation of ischemia-reperfusion injuryinduced tissue inflammatory processes and resultant recipient adaptive immune response to donor tissue during allogeneic renal transplantation. Nitric Oxide. 2013;31(2):S60-1. DOI: 10.1016/j. niox.2013.06.119.

6. Hassoun H, Grigoryev DN, Lie M, Liu M, Cheadle C, Tuder RM, et al. Ischemic acute kidney injury induces a distant organ functional and genomic response distinguishable from bilateral nephrectomy. Am J Physiol Renal Physiol. 2007;290:30-40. PMID: 17327501.

7. Hsieh CC, Hsieh SC, Chiu JH, Wu YL. Protective effects of $\mathrm{N}$-acetylcysteine and a prostaglandin E1 analog, Alprostadil, against hepatic ischemia/reperfusion injury in Rats. J Tradit Complement Med. 2014;4(1):64-71. DOI: 10.4103/22254110.124351 .

8. Uysal AI, Ocmen E, Akan M, Ozkardesler S, Ergur BU, Guneli E, Kume T, Koca U, Togrul BU. The effects of remote ischemic preconditioning and $\mathrm{N}$-acetylcysteine with remote ischemic preconditioning in rat hepatic ischemia reperfusion injury model. Biomed Research Inten. 2014;ID 892704,6 pages. PMDI: 24511549.

9. Robinson SM, Saif R, Sen G, French JJ, Jaques BC, Charnley RM, Manas DM, White SA. N-acetylsysteine administration does not improve patient outcome after liver resection. HPB (Oxford). 2013;15(6):457-62. DOI: 10.1111/hpb.12005.

10. Lee EJS, Silva SM, Simões MJ, Montero EFS. Effect of $\mathrm{N}$-acetylcysteine in liver ischemia-reperfusion injury after $30 \%$ hepatectomy in mice. Acta Cir Bras. 2012;27(4): 346-9. DOI: 10.1590/S0102-86502012000400011.

11. Kerr JF, Wyllie AH, Currie AR. Apoptosis: a basic biological phenomenon with wide-ranging implications in tissue kinetics. Br J Cancer. 1972;26:239-57. PMID: 4561027.

12. D'Amico F, Vitale A, Piovan D, Bertacco A, Ramirez Morales R, Chiara Frigo A, Bassi D, Bonsignori B, Grigeri E, Valmasoni M, Garbo G, Lodo E, D'Amico FE, Scopelliti M, Carraro A, Gambato M, Brolese A, Zanus G, Neri D, Cillo U. Use of $\mathrm{N}$-acetylcysteine during liver procurement: a prospective randomized controlled study. Liver Transpl. 2013;19(2):135-44. DOI: $10.1002 / 1$ t. 23527 .
13. Zhu J, Wang S, Bie P, Li X, Zhang Y, Xiong Y, Liang H, Ma Z, Li K, Dong, J. Apoptosis and regeneration of sinusoidal endothelial cells after extended cold preservation and transplantation of rat liver. Transplantation. 2007;84:1483-91. DOI: 10.1097/01. tp.0000290188.38041.f1.

14. Doustar Y, Mohajeri D, Garjani A, Mousavi G, Ghramaleki MN. Protective Effect of Metformin on Cardiomyocytes IschemiaReperfusion (IR) Induced Apoptosis in Rats. Journal of Animal and Veterinary Advances. 2012;11(1):108-12. DOI: 10.3923/ java.2012.108.112

15. Lasham MM, Lamine AJ, Dhouib IB, Bouzid K, Annabi A, Belhadjhmida N, Ahmed MB, El Fazaa S, Abdelmoula J, Gharbi N. Antioxidant and anti-inflammatory effects of $\mathrm{N}$-acetylcysteine against malathion-induced liver damages and immunotoxicity in rats. Life Sci. 2014;107(1-2):50-8. DOI: 10.1016/j.1fs.2014.04.033.

16. Feldmann G. Liver apoptosis. Gastroenterol Clin Biol. 2006;30(4):533-45. DOI: GCB-04-2006-30-4-0399-8320-101019200517795.

17. Didoné EC, Cerski CK, Kalil AN. N-acetylcysteine decreases hepatic congestion in the ischemia-reperfusion injury - experimental study. Rev Col Bras Cir. 2002;29(4):191-6. ISSN: 01006991. DOI: 10.1590/S0100-69912002000400002.

18. Guarrera JV, Niaz KA. Liver preservation: is there anything yet? Curr Opin Organ Transplant. 2008;13:148-54. DOI: 10.1097/ MOT.0b013e3282f63930.

\section{Correspondence:}

Edna Frasson de Souza Montero

Alameda Espada, 134 - Res. Onze - Alphaville

CEP 06540-395 - Santana de Parnaíba - São Paulo - Brazil

E-mail: edna.montero@gmail.com

Financial source: São Paulo Research Foundation (FAPESP)

${ }^{1}$ Research performed at Operative Technique and Experimental Surgery Division, Department of Surgery, UNIFESP, Brazil. 